\title{
Article
}

\section{The Comparative Inclusive Human Development of Globalisation in Africa}

\author{
Asongu, Simplice A. and Nwachukwu, Jacinta Chikaodi \\ Available at http://clok.uclan.ac.uk/24916/ \\ Asongu, Simplice A. and Nwachukwu, Jacinta Chikaodi ORCID: 0000-0003- \\ 2987-9242 (2017) The Comparative Inclusive Human Development of \\ Globalisation in Africa. Social Indicators Research, 134 (3). pp. 1027-1050. \\ ISSN 0303-8300
}

It is advisable to refer to the publisher's version if you intend to cite from the work. http://dx.doi.org/10.1007/s11205-016-1467-2

For more information about UCLan's research in this area go to http://www.uclan.ac.uk/researchgroups/ and search for < name of research Group>.

For information about Research generally at UCLan please go to http://www.uclan.ac.uk/research/

All outputs in CLoK are protected by Intellectual Property Rights law, including Copyright law. Copyright, IPR and Moral Rights for the works on this site are retained by the individual authors and/or other copyright owners. Terms and conditions for use of this material are defined in the policies page.

\section{CLoK}

Central Lancashire online Knowledge www.clok.uclan.ac.uk

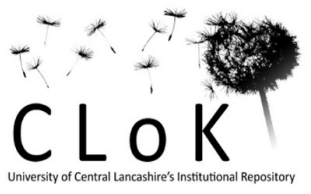




\section{A G D I Working Paper}

\section{WP/16/038}

The Comparative Inclusive Human Development of Globalisation in Africa

Forthcoming: Social Indicators Research

Simplice A. Asongu

African Governance and Development Institute, P.O. Box 8413 Yaoundé, Cameroon.

E-mail: asongusimplice@yahoo.com /asongus@afridev.org

Jacinta C. Nwachukwu

School of Economics, Finance and Accounting,

Faculty of Business and Law,

Coventry University

Priory Street, Coventry, CV1 5FB, UK

Email: jacinta.nwachukwu@coventry.ac.uk 
Research Department

\title{
The Comparative Inclusive Human Development of Globalisation in Africa
}

\author{
Simplice A. Asongu \& Jacinta C. Nwachukwu
}

March 2016

\begin{abstract}
This study examines the impact of globalisation on inclusive human development in 51 African countries for the period 1996-2011 with particular emphasis on income levels (low income versus middle income), legal origins (English common law versus French civil law), resource wealth (oil-rich versus oil-poor), landlockedness (landlocked versus unlandlocked), religious domination (Christianity versus Islam) and political stability (stable versus unstable). The empirical evidence is based on instrumental variable panel Fixed effects and Tobit regressions in order to control for the unobserved heteroegeneity and limited range in the dependent variable. Political, economic, social and general globalisation variables are used. Six main hypotheses are investigated. The findings broadly show that middle income, English common law, oil-poor, unlandlocked, Christian-oriented and politically-stable countries are associated with comparatively higher levels of globalisation-driven inclusive human development. Puzzling findings are elucidated and policy implications discussed.
\end{abstract}

JEL Classification: E60; F40; F59; D60; O55

Keywords: Globalisation; inequality; inclusive development; Africa

\section{Introduction}

There are three principal motivations for investigating the comparative economics of globalisation on inclusive human development in Africa. They are: (i) recent inclusive development trends and (ii) debates surrounding the relationship between inclusive development and (iii) the contemporary relevance of making globalisation more inclusive and the need to present findings with more targeted policy implications that are based on some 
fundamental features of inclusive human development. We engage these points in chronological order.

First, a World Bank report on attainment of Millennium Development Goal (MDGs) targets of extreme poverty has recently shown that with the exception of the African continent, poverty has been decreasing in all regions of the world. In sub-Saharan Africa, $45 \%$ of countries were still considerably off-track from the achieving the MDGs extreme poverty targets (see World Bank, 2015). This dismal evidence on Africa substantially contrasts with statistics that the continent has been enjoying more than two decades of resurgence in growth that began in the mid 1990s (see Fosu, 2015a). It also sharply contrasts with optimistic discourses on and narrative of 'Africa rising' (Leautier, 2012) as well as premature conclusions that all African countries, with the exception of the Democratic Republic of Congo, had reached the MDGs extreme poverty target by the end of 2014 or one year ahead of time (Pinkivskiy \& Sala-i-Martin, 2014). Obeng-Odoom (2015) has attributed the contrast to policies of neoliberalism and globalisation that are more focused on increasing the relevance of capital accumulation and the neoliberal ideology, with less concern to more fundamental ethnical issues like environmental degradation and inequality.

Second, there is yet no consensus in the literature on the appeals of globalisation in development outcomes. Whereas growing financial and economic instability has been documented as resulting from increasing globalisation, there is also some evidence on the potential rewards of globalisation in terms of allocation efficiency and international risk sharing (Price \& Elu, 2014; Kose et al., 2006, 2011; Asongu, 2014a). The contemporary economic landscape has been characterised by two main trends in the past thirty years, notably: growing inequality and increasing globalisation (Azzimonti et al., 2014). Within the same period, the concern about exclusive development has been an increasing concern in developed nations (Atkinson et al., 2011; Piketty, 2014), a broad sample of developing countries (Mlachila et al., 2014; Mthuli et al., 2014; Fosu, 2010a) as well as in African countries (Fosu, 2008, 2009, 2010b, 2010c).

Third, the sustainable development agenda in the post-2015 era is articulated by a policy syndrome of an increasingly globalised world that is less inclusive in terms of human development (UN, 2013, pp. 7-13). Accordingly, the ineluctable globalisation process was initiated with the promise of economic development (Tchamyou, 2015). Unfortunately, this phenomenon is threatening to disfigure the human face because it endangers the progress of nations and people, it advocates market over governments and self interest over altruism. It is 
therefore unsurprising that public support for the phenomenon is decreasing both in developing and developed nations, with fervent explorations of alternatives to the morallydisturbing side of the capitalism-driven globalisation (Asongu, 2013; Kenneth \& Himes, 2008; Stiglitz, 2007).

In the light of the above, this inquiry extends the literature that has been responding to the World Bank report on the African continent's extreme poverty tragedy. We complement the existing literature by investigating the impact of globalisation on inclusive development with particular emphasis on some fundamental characteristics of comparative inclusive human development. From intuition, findings from comparative economic development are more likely to produce more targeted policy implications. Hence, in order to provide room for more policy options, the dataset is disaggregated into the fundamental characteristics of human development based on: income levels (low income and middle income); legal origins (English Common law and French Civil law); religious domination (Christianity and Islam); openness to sea (landlocked and unlandlocked); resource-wealth (oil-rich and oil-poor) and political stability (stable and unstable).

The existing literature (on responses to the World Bank report) which this inquiry extends can be discussed in three main strands: (i) novel paradigms of development and insights into Africa's resurgence in growth; (ii) the reinvention of development assistance for the purposes of inclusive development and (iii) the role of globalisation in inclusive human development. In the first strand, Fosu (2015bc) edited a book with numerous articles that are focused on examining whether the recent growth experience by African counties is myth or reality. Kuada (2015) has also designed a book that elicits Africa's extreme poverty tragedy. The author recommends a paradigm shift from 'strong economics' (or debates on neoliberal policies) to 'soft economics' (or human capability development) as a mechanism to understanding the recent trends in poverty experienced across the board. In the second part, the proposals of Kuada (2015) are consistent with a stream of the literature that is suggesting some mechanisms by which foreign aid can be reinvented for the purposes of enhancing sustainable development, greater employment and less poverty (see Jones et al., 2015; Asongu \& Nwachukwu, 2016a; Simpasa et al., 2015; Page \& Söderbom, 2015; Asongu, 2015a; Jones $\&$ Tarp, 2015; Fields, 2015; Page \& Shimeles, 2015). The third element which is closest to the present study has focused on the link between globalisation and inclusive development, with a notable study by Azzimonti et al. (2014) which has theorized that globalisation-fuelled debts are a fundamental cause of exclusive development in advanced economies. The 
empirical validity of the theory has been partially confirmed in Africa (see Asongu et al., 2015).

The rest of the study is structured as follows. The theoretical underpinnings and testable hypotheses are presented in Section 2. The data and methodology are discussed in Section 3 while Section 4 covers the presentation of results and policy implications. Section 5 concludes with future research directions.

\section{Theoretical underpinnings and testable hypotheses}

\subsection{Theoretical underpinnings}

There are two main theoretical underpinnings on the relationship between globalisation and inclusive development: the hegemony and neoliberal perspectives (see Tsai, 2006). According to hegemonic perspective, the phenomenon of globalisation is a surreptitious project that aims to create a new order in the world which is dictated by global forces like industrial countries and powerful financial institutions. According to this thought, a main goal of globalisation is to ease capital accumulation and extend the benefits of openness from trade in commodities (good and services) to trade in financial assets. Authors in the stream foresee 'a world-wide crisis of living standards for labor', given that the process of capital liberalisation has been shouldered by the working class because 'technological change and economic reconversion endemic to capitalist development has generated an enormous growing pool of surplus labor, an industrial reserve army with incomes at or below the level of subsistence' (Petras \& Veltmeyer, 2001, p. 24).

There is another side to the hegemonic perspective maintaining that the modes of production by policies of neoliberalism are oriented towards a dynamic production process that undermines mechanisms of redistribution that were advocated by Keynesian Social democracy. According to Smart (2003), the phenomenon of globalisation is more friendly to the quest for private interest, while paying less attention to more ethical concerns like inclusive development. In essence, the process of distributing the rewards accruing from globalisation is skewed to the advantage of the wealthy segments of the population who are already in advantageous positions from socio-economic standpoints (Scholte, 2000). The perspective of Scholte is shared by Sirgy et al (2004), though in a less radical tone.

The neoliberal or second school disputes that globalisation is an instrument of 'creative destruction' in the view that technological innovation, cross-border investment and global trade improve production efficiency and make considerable progress despite falling 
wages for unskilled workers and job substitution. The inconveniences of globalisation are managed by requesting unskilled workers to acquire new skills in order to benefit from openness. From the perspective of Grennes (2003), such rewards can benefit the masses if 'supply and demand' affects the market of labour.

\subsection{Testable hypotheses for comparative human development}

We discuss the testable hypotheses for the comparative inclusive human development in terms of income levels, legal origins, religious domination, openness to sea, natural resources and political stability. Recent inclusive development literature has employed these fundamental characteristics for comparative development (see Mlachila et al., 2014).

First, compared to middle income countries, low income countries are more likely to be associated with less effective institutions that oversee the equitable distribution of the fruits of economic prosperity. There are two principal motivations for the positive association between income levels and higher inclusive development. On the one hand, higher income provides more avenues for social mobility and employment. On the other, institutions have been documented to positively influence growth quality (Mlachila et al., 2014; Fosu, 2015bc), especially in terms of enhancing standards of living by means of better management of resources (Fosu, 2013ab; Fonchingong, 2014; Anyanwu \& Erhijakpor, 2014) and consolidation of the foundations for social change (Efobi, 2015).

Hypothesis 1: Compared to low income countries, middle income countries have higher levels of inclusive human development.

Second, legal origins are crucial in contemporary comparative development (see La Porta et al., 1998, 1999). The African continent is therefore not an exception to this assertion (see Agbor, 2015). In essence, French Civil law and English Common law countries differ in how legal origins affect institutions because of differences in political and adaptability mechanisms (see Beck et al., 2003). In Africa, legal origins have been recently documented to affect welfare (Asongu, 2015). According to the description, whereas countries with French Civil Law place more emphasis on the power of the state, English Common Law is more concerned with consolidating private property rights which is likely to improve conditions that reduce economic vulnerability and increase the social mobility needed for inclusive development. In essence, the institutional web of formal rules, informal norms and characteristics of enforcement affect economic vulnerability and social mobility within a country. 
Hypothesis 2: English Common Law countries have higher levels of inclusive human development compared to their French Civil Law counterparts.

Third, from intuition, politically stable countries are more likely to create better conditions for inclusive development compared to their politically unstable counterparts. The insight is consistent with Beegle et al. (2016, p.10) who have concluded that fragility is associated with significantly slower poverty reduction in Africa. While this perception is sound, it has to be ascertained with empirical justification.

Hypothesis 3: Politically stable countries are associated with higher levels of inclusive development, relative to politically unstable countries.

Fourth, whereas the prospect of higher inclusive development in resource-rich countries is consistent with the hypothesis on income-wealth, there are also strong reasons to suggest that countries that have acknowledged scarcity in natural resources have focused more on human capability development as means to achieving growth and inclusive development (Fosu, 2013b; America, 2013; Amavilah, 2015). This assertion is in line with the Kuada (2015) paradigm on 'soft economics' as a means to understanding Africa's poverty tragedy.

Hypothesis 4: Resource-poor countries are associated with higher levels of inclusive development, compared to their resource-wealthy counterparts

Fifth, landlockedness has institutional and economic (Arvis et al., 2007) costs which are very likely to influence economic prosperity, economic vulnerability, social mobility and the distribution of the fruits of economic prosperity. In essence, the institutional cost, inter alia; should intuitively be connected with less economic governance: the formulation and implementation of effective policies that deliver public commodities for inclusive development.

Hypothesis 5: Landlocked countries are associated with lower levels of inclusive development compared to countries that are opened to the sea.

We also use religious domination as a fundamental characteristic of comparative inclusive human development. This is essentially because the basis for religious supremacy is founded on the supposition that solidarity affects inclusiveness. The two dominant models of such solidarity in Africa are Christianity and Islam. Compared to countries with Islam- 
domination that are traditionally more conservative with regard to neoliberalism, Christiandominated countries are less conformist with respect to the neoliberal ideology. Such could influence the choice of neoliberal policies in globalisation for inclusive human development (Roudometof, 2014).

Hypothesis 6: Christian-dominated countries are associated with higher levels of inclusive development, compared to their Islam-oriented counterparts.

In the light of the above, the selected fundamental characteristics have some influence on the adoption of neoliberal and/or globalisation policies which ultimately affect economic prosperity and inclusive human development.

\section{Data and Methodology}

\subsection{Data}

In this study, we assess a panel of 51 African countries with data for the period 19962011 from Dreher et al. (2010); African Development Indicators of the World Bank and the United Nations Development Program (UNDP). The sampled countries and periodicity are due to data availability constraints. Consistent with recent African inclusive development literature (Asongu \& Nwachukwu, 2016b), the inequality adjusted human development index (IHDI) from the UNDP is used as a proxy for inclusive development. The IHDI is the national average of achievements in three main areas, namely: (i) knowledge; (ii) health and long life and (iii) reasonable standards of living. In addition to accounting for average rewards in terms of health, education and income, the IHDI also accounts for the distribution of underlying achievements among the population by controlling for mean values of each dimension with regards to inequality.

Globalisation indicators from Dreher et al (2010) are the independent variables of interest. These are economic, social, political and general globalisation. The control variables which are from African Development Indicators are also selected in accordance with the literature on inclusive development (Mishra et al., 2011; Anand et al., 2012; Seneviratne \& Sun, 2013; Mlachila et al., 2014; Asongu \& Nwachukwu, 2016c). The control variables are: public investment, GDP growth, inflation and development assistance. Economic prosperity is expected to positively influence inclusive development (Mlachila et al., 2014). While development assistance has been shown to negatively affect inclusive human development when 'types of aid' are considered, both positive and negative impacts are apparent (Asongu 
\& Nwachukwu, 2016a). Public investment could either have a negative or positive effect contingent on whether funds are mismanaged and/or if corrupt channels are involved in processes of disbursing funds for inclusive development goals. Chaotic inflation reduces inclusive development because compared to the rich, whose purchasing power is not substantially affected, the poor bore a greater burden in terms of decreasing purchasing power. Appendix 1 provides the definitions of variables while Appendix 2 discloses the summary statistics. The correlation matrix is presented in Appendix 3.

The awareness for the choice of fundamental characteristics for comparative human development has been covered in Section $2^{1}$. The selection criteria for these fundamental features are consistent with recent literature on inclusive growth in developing countries (Mlachila et al., 2014, p. 13) and African human development (Asongu, 2014c, p.339). Accordingly, classification of countries in terms of legal traditions is from La Porta et al. (2008, p 289) and categorisation of nations by income levels is consistent with Asongu (2014b, p. 364) $)^{2}$ on the World Bank classification. Resource-wealth is exclusively based on oil-dominated exports representing at least 30 percent of GDP for at least a decade in the sampled periodicity. Landlocked and unlandlocked countries are apparent from an African map while classification of religious domination is from the Central Intelligence Agency (CIA) World Fact Book (CIA, 2011). Politically unstable countries are those that have experienced political instability/violence for at least half of the sampled periodicity. The categorisation of countries is presented in Appendix 4.

\subsection{Methodology}

Consistent with recent inclusive development literature that has used the IHDI, we adopt both Tobit and Fixed effects (FE) regressions in order to respectively account for the limited range in the dependent variable and the unobserved heterogeneity. Hence, instrumental variable (IV) FE and Tobit estimations are used. Contrary to the underlying study that has also employed the Generalized Method of Moments (GMM) to control for persistence in inclusive human development, we cannot employ the GMM here because $\mathrm{T}>\mathrm{N}$ for some fundamental characteristics. It is important to note that a basic requirement for the

\footnotetext{
${ }^{1}$ It is important to note that, whereas the motivations for the choice of fundamental characteristics are the testable hypotheses that are derived hereafter in Section 2, the criteria for the selection of fundamental characteristics are now engaged.

2 There are four main World Bank income groups: (i) high income, \$12,276 or more; (ii) upper middle income, $\$ 3,976-\$ 12,275$; (iii) lower middle income, $\$ 1,006-\$ 3,975$ and (iv) low income, $\$ 1,005$ or less.
} 
application of GMM is $\mathrm{T}<\mathrm{N}$. Accordingly, whereas $\mathrm{T}$ is equal to 16 years (or 1996-2011) some panels encompass countries that are less than $\mathrm{T}$. These include inter alia: landlocked and conflict-affected countries.

The concern of simultaneity and/or reverse causality is addressed by instrumenting the globalisation variables of interest with their first lags. This instrumentation process is summarised in Eq. (1) below.

$G_{i, t}=\alpha+\delta_{j}\left(G_{i, t-1}\right)+\varepsilon_{i, t}$

Where: $G_{i, t}$, is a globalisation indicator of country $i$ at period $t, G_{i, t-1}$, represents globalisation in country $i$ at period $t-1, \alpha$ is a constant and $\varepsilon_{i, t}$ the error term. The instrumentation procedure consists of regressing the independent variables of interest on their first lags and then saving the fitted values that are subsequently used as the main independent variables in Eq. (2) and Eq. (4) for the FE and Tobit regressions respectively. The specifications are Heteroscedasticity and Autocorrelation Consistent (HAC) in standard errors.

The panel FE model is presented as follows in Eq. (2)

$$
I H D_{i, t}=\partial_{0}+\partial_{1} I V G_{i, t}+\sum_{h=1}^{4} \omega_{h} W_{h, i, t-\tau}+\eta_{i}+\varepsilon_{i, t}
$$

Where: $I H D_{i, t}$ is inclusive human development of country $i$ at period $t ; \partial$ is a constant; $I V G$, instrumented globalisation; $W$ is the vector of control variables (GDP growth, Foreign aid, Public investment and Inflation); $\eta_{i}$ is the country-specific effect and $\varepsilon_{i, t}$ the error term.

Given that the range of the IHDI is theoretically between 0 and 1 ( 0.127 to 0.809 as in this study), Ordinary Least Squares (OLS) may be inappropriate. In order to control for the limited range in the dependent variable, Tobit models are a good fit (Kumbhakar \& Lovell, 2000; Koetter et al., 2008; Ariss, 2010; Coccorese \& Pellecchia, 2010). In accordance with McDonald (2009) and Coccorese and Pellecchia if there are no observations of either 0 or 1 for the dependent variable (which is the case with the IHDI in this study), estimating by a double-censored Tobit model is similar to estimating by a linear regression model because the two likelihood functions coincide. Therefore, the logistic regression associated with the Tobit model is as follows in Eq. (3): 
$I H D_{i t}=\frac{\exp \left(x_{i t}^{\prime} \beta\right)}{1+\exp \left(x_{i t}^{\prime} \beta\right)}+\phi_{i t}$

where $x_{i t}$ is the same vector of regressors used in the Tobit model, $\beta$ is the vector of parameters and $\phi_{i t}$ is independently and identically distributed (iid) with mean zero and variance $\sigma_{\phi}^{2}$ variance.

The standard Tobit model (Tobin, 1958; Carsun \& Sun, 2007) is as follows in Eq. (4):

$$
y_{i, t}^{*}=\alpha_{0}+\beta X_{i, t}+\varepsilon_{i, t}
$$

where $y_{i, t}^{*}$ is a latent response variable, $X_{i, t}$ is an observed $1 \times k$ vector of explanatory variables and $\varepsilon_{i, t} \approx$ i.i.d. $\mathrm{N}(0, \sigma 2)$ and is an independent variable of $X_{i, t}$. Instead of observing $y_{i, t}^{*}$, we observe $y_{i, t}$ as follows in Eq. (5):

$$
y_{i, t}=\left\{\begin{array}{c}
y_{i, t}^{*} \text { if } y_{i, t}^{*}>\gamma \\
0, \text { if } y_{i, t}^{*} \leq \gamma,
\end{array}\right.
$$

where $\gamma$ is a non stochastic constant. In other words, the value of $y_{i, t}^{*}$ is missing when it is less than or equal to $\gamma$.

\section{Empirical Results}

\subsection{Presentation of baseline results}

The baseline results are presented in Table 1. The left-hand-side presents instrumental variable (IV) FE regressions whereas the right-hand-side shows findings from corresponding instrumental variable (IV) Tobit estimations. It is apparent that globalisation consistently improves inclusive development, with the lowest effect from political globalisation and highest impact from general globalisation. The significant control variables display the expected signs.

Table 2 and Table 3 respectively decompose the IV FE and Tobit regressions into fundamental characteristics. For either table, Panel A, Panel B, Panel C and Panel D respectively present findings related to political, economic, social and general globalisation. Based on the findings in Table 2, the investigated hypotheses are confirmed in the following order of comparative significance: political globalisation; general governance; economic globalisation and social globalisation. In the Tobit regressions, the tested hypotheses are 
overwhelmingly confirmed. In addition, the panel on social globalisation (or Panel C) which did not show overwhelming comparative significance in the FE regressions (see Table 2) now has many comparative pairs which are significant. The significant control variables have the expected signs.

Table 1: Baseline Instrumental Variable Fixed Effects and Tobit regressions

\begin{tabular}{|c|c|c|c|c|c|c|c|c|}
\hline \multirow[b]{3}{*}{ Constant } & \multicolumn{8}{|c|}{ Dependent variable: Inequality Adjusted Human Development Index (IHDI) } \\
\hline & \multicolumn{4}{|c|}{ Fixed Effects } & \multicolumn{4}{|c|}{ Tobit } \\
\hline & $\begin{array}{l}1.443 * * * \\
(0.000)\end{array}$ & $\begin{array}{l}1.513 * * * \\
(0.000)\end{array}$ & $\begin{array}{l}1.502 * * * \\
(0.000)\end{array}$ & $\begin{array}{l}1.379 * * * \\
(0.000)\end{array}$ & $\begin{array}{l}1.304 \\
(0.397)\end{array}$ & $\begin{array}{l}-3.840 * * \\
(0.011)\end{array}$ & $\begin{array}{l}-1.543 \\
(0.252)\end{array}$ & $\begin{array}{l}-6.888 * * * \\
(0.000)\end{array}$ \\
\hline Political Glob. (IV) & $\begin{array}{l}0.003 * * * \\
(0.000)\end{array}$ & --- & --- & -- & $\begin{array}{l}0.042 * * \\
(0.042)\end{array}$ & --- & --- & -- \\
\hline Economic Glob.(IV) & --- & $\begin{array}{l}0.004 * * \\
(0.013)\end{array}$ & --- & --- & --- & $\begin{array}{l}\mathbf{0 . 1 7 7} * * * \\
(\mathbf{0 . 0 0 0 )}\end{array}$ & --- & --- \\
\hline Social Glob. (IV) & --- & --- & $\begin{array}{l}0.005 * * \\
(0.038)\end{array}$ & --- & --- & -- & $\begin{array}{l}\mathbf{0 . 1 7 0} * * * * \\
(\mathbf{0 . 0 0 0 )}\end{array}$ & --- \\
\hline Globalisation(Glob) (IV) & --- & --- & -- & $\begin{array}{l}0.006 * * * \\
(0.000)\end{array}$ & --- & --- & -- & $\begin{array}{l}0.242 * * * * \\
(0.000)\end{array}$ \\
\hline GDP growth & $\begin{array}{l}0.001 \\
(0.573)\end{array}$ & $\begin{array}{l}0.0008 \\
(0.735)\end{array}$ & $\begin{array}{l}0.001 \\
(0.574)\end{array}$ & $\begin{array}{l}0.0008 \\
(0.722)\end{array}$ & $\begin{array}{l}0.056 \\
(0.531)\end{array}$ & $\begin{array}{l}0.048 \\
(0.585)\end{array}$ & $\begin{array}{l}0.054 \\
(0.536)\end{array}$ & $\begin{array}{l}0.039 \\
(0.654)\end{array}$ \\
\hline Foreign aid & $\begin{array}{l}0.00005 \\
(0.971)\end{array}$ & $\begin{array}{l}-0.0001 \\
(0.901)\end{array}$ & $\begin{array}{l}-0.0003 \\
(0.803)\end{array}$ & $\begin{array}{l}-0.0002 \\
(0.877)\end{array}$ & $\begin{array}{l}-0.112 * * * * \\
(0.005)\end{array}$ & $\begin{array}{l}-0.040 \\
(0.327)\end{array}$ & $\begin{array}{l}-0.026 \\
(0.537)\end{array}$ & $\begin{array}{l}-0.005 \\
(0.898)\end{array}$ \\
\hline Public Investment & $\begin{array}{l}-0.001 \\
(0.635)\end{array}$ & $\begin{array}{l}-0.0003 \\
(0.924)\end{array}$ & $\begin{array}{l}-0.0003 \\
(0.930)\end{array}$ & $\begin{array}{l}-0.001 \\
(0.657)\end{array}$ & $\begin{array}{l}-0.218 * * \\
(0.020)\end{array}$ & $\begin{array}{l}-0.293 * * * \\
(0.003)\end{array}$ & $\begin{array}{l}-\mathbf{0 . 2 4 5} * * * * \\
(0.008)\end{array}$ & $\begin{array}{l}-0.279 * * * \\
(0.002)\end{array}$ \\
\hline Inflation & $\begin{array}{l}-0.000 \\
(0.679)\end{array}$ & $\begin{array}{l}-0.000 \\
(0.735)\end{array}$ & $\begin{array}{l}-0.000 \\
(0.704)\end{array}$ & $\begin{array}{l}-0.000 \\
(0.681)\end{array}$ & $\begin{array}{l}-0.000 \\
(0.676)\end{array}$ & $\begin{array}{l}-0.0001 \\
(0.651)\end{array}$ & $\begin{array}{l}-0.0001 \\
(0.626)\end{array}$ & $\begin{array}{l}-0.0001 \\
(0.562)\end{array}$ \\
\hline Adjusted R²(within) & 0.031 & 0.016 & 0.011 & 0.030 & --- & --- & --- & --- \\
\hline Fisher & $2.71 * *$ & 1.36 & 0.99 & $2.62 * *$ & --- & --- & --- & --- \\
\hline LR Chi-Square & --- & --- & --- & --- & $22.15 * * *$ & $52.88 * * *$ & $42.19 * * *$ & $55.58 * * *$ \\
\hline Log Likelihood & --- & --- & --- & --- & -1532.431 & -1484.800 & -1522.408 & -1515.714 \\
\hline Countries & 35 & 33 & 35 & 35 & --- & --- & --- & --- \\
\hline Observations & 453 & 442 & 453 & 453 & 453 & 442 & 453 & 453 \\
\hline
\end{tabular}

$*, * * * * *$ : significance levels of $10 \%, 5 \%$ and $1 \%$ respectively. IV: Instrumented Variable.

\section{2: Comparative economics with Instrumental Variable Fixed Effects}

\begin{tabular}{|c|c|c|c|c|c|c|c|c|c|c|c|c|}
\hline & \multicolumn{12}{|c|}{ Panel A: Political globalisation } \\
\hline & \multicolumn{2}{|c|}{ Income levels } & \multicolumn{2}{|c|}{ Legal origins } & \multicolumn{2}{|l|}{ Religion } & \multicolumn{2}{|c|}{ Openness to sea } & \multicolumn{2}{|c|}{ Oil exports } & \multicolumn{2}{|c|}{ Political stability } \\
\hline & LI & MI & Eng. & Frch. & Christ. & Islam & Open & Closed & Oil & Nonoil & Stable & Unstable \\
\hline Constant & $\begin{array}{l}0.242 * * * \\
(0.000)\end{array}$ & $\begin{array}{l}3.128 * * * \\
(0.000)\end{array}$ & $\begin{array}{l}3.546 * * * \\
(0.000)\end{array}$ & $\begin{array}{l}0.283 * * * \\
(0.000)\end{array}$ & $\begin{array}{l}2.010 * * * * \\
(0.000)\end{array}$ & $\begin{array}{l}0.291 * * * \\
(0.000)\end{array}$ & $\begin{array}{l}2.331 * * * \\
(\mathbf{0 . 0 0 0 )}\end{array}$ & $\begin{array}{l}0.249 * * * \\
(0.000)\end{array}$ & $\begin{array}{l}0.407 * * * \\
(0.000)\end{array}$ & $\begin{array}{l}1.649 * * * \\
(0.000)\end{array}$ & $\begin{array}{l}1.753 * * * \\
(0.000)\end{array}$ & $\begin{array}{l}0.241 * * * \\
(0.000)\end{array}$ \\
\hline Political Glob (IV) & $\begin{array}{l}0.002 * * * \\
(0.000)\end{array}$ & $\begin{array}{l}0.006 * * \\
(0.019)\end{array}$ & $\begin{array}{l}\text { 0.006** } \\
(\mathbf{0 . 0 3 3 )}\end{array}$ & $\begin{array}{l}0.002 * * * \\
(0.000)\end{array}$ & $\begin{array}{l}0.004 * * * \\
(0.004)\end{array}$ & $\begin{array}{l}0.002 * * * \\
(0.000)\end{array}$ & $\begin{array}{l}0.005 * * * \\
(0.009)\end{array}$ & $\begin{array}{l}0.002 * * * \\
(0.000)\end{array}$ & $\begin{array}{l}\mathbf{0 . 0 0 1} * * * \\
(\mathbf{0 . 0 0 0 )}\end{array}$ & $\begin{array}{l}0.004 * * * \\
(0.001)\end{array}$ & $\begin{array}{l}0.004 * * * \\
(0.001)\end{array}$ & $\begin{array}{l}\mathbf{0 . 0 0 1} * * * \\
(\mathbf{0 . 0 0 0 )}\end{array}$ \\
\hline GDP growth & $\begin{array}{l}0.001 * * * \\
(0.001)\end{array}$ & $\begin{array}{l}0.004 \\
(0.623)\end{array}$ & $\begin{array}{l}0.002 \\
(0.753)\end{array}$ & $\begin{array}{l}0.0004 \\
(0.110)\end{array}$ & $\begin{array}{l}0.001 \\
(0.700)\end{array}$ & $\begin{array}{l}\text { 0.001** } \\
(\mathbf{0 . 0 1 8 )}\end{array}$ & $\begin{array}{l}0.001 \\
(0.824)\end{array}$ & $\begin{array}{l}0.001 * * * \\
(0.001)\end{array}$ & $\begin{array}{l}0.0009 * * \\
(0.010)\end{array}$ & $\begin{array}{l}0.001 \\
(0.724)\end{array}$ & $\begin{array}{l}0.0001 \\
(0.961)\end{array}$ & $\begin{array}{l}\text { 0.001**** } \\
(\mathbf{0 . 0 0 0 )}\end{array}$ \\
\hline Foreign aid & $\begin{array}{l}-0.0001 \\
(0.302)\end{array}$ & $\begin{array}{l}0.001 \\
(0.940)\end{array}$ & $\begin{array}{l}0.006 \\
(0.556)\end{array}$ & $\begin{array}{l}-0.0002 \\
(0.186)\end{array}$ & $\begin{array}{l}0.0001 \\
(0.925)\end{array}$ & $\begin{array}{l}0.002 \\
(0.533)\end{array}$ & $\begin{array}{l}-0.0005 \\
(0.835)\end{array}$ & $\begin{array}{l}0.0003 \\
(0.325)\end{array}$ & $\begin{array}{l}-0.001 * \\
(0.051)\end{array}$ & $\begin{array}{l}0.0002 \\
(0.875)\end{array}$ & $\begin{array}{l}0.0004 \\
(0.881)\end{array}$ & $\begin{array}{l}-0.0003 \\
(0.304)\end{array}$ \\
\hline Public Investment & $\begin{array}{l}0.0008 \\
(0.204)\end{array}$ & $\begin{array}{l}-0.003 \\
(0.625)\end{array}$ & $\begin{array}{l}-0.010 \\
(0.404)\end{array}$ & $\begin{array}{l}0.002 * * * \\
(0.000)\end{array}$ & $\begin{array}{l}-0.005 \\
(0.400)\end{array}$ & $\begin{array}{l}0.0001 \\
(0.580)\end{array}$ & $\begin{array}{l}-0.003 \\
(0.616)\end{array}$ & $\begin{array}{l}0.0002 \\
(0.739)\end{array}$ & $\begin{array}{l}\mathbf{0 . 0 0 2} * * * \\
(\mathbf{0 . 0 0 0 )}\end{array}$ & $\begin{array}{l}-0.004 \\
(0.372)\end{array}$ & $\begin{array}{l}-0.002 \\
(0.620)\end{array}$ & $\begin{array}{l}0.002 * \\
(0.096)\end{array}$ \\
\hline Inflation & $\begin{array}{l}-0.000 * * * \\
(0.002)\end{array}$ & -0.004 & -0.000 & -0.000 & -0.000 & $\begin{array}{l}0.0001 \\
(0.580)\end{array}$ & $\begin{array}{l}-0.0001 \\
(0.803)\end{array}$ & $\begin{array}{l}-0.000 * * * \\
(0.003)\end{array}$ & $\begin{array}{l}-0.0003 \\
(0.299)\end{array}$ & $\begin{array}{l}-0.000 \\
(0.680)\end{array}$ & $\begin{array}{l}-0.001 \\
(0.619)\end{array}$ & $\begin{array}{l}\mathbf{0 . 0 0 0 * * *} \\
(\mathbf{0 . 0 0 2})\end{array}$ \\
\hline Adjusted $\mathrm{R}^{2}$ (within) & 0.523 & 0.039 & 0.036 & 0.591 & 0.030 & 0.527 & 0.030 & 0.521 & 0.538 & 0.034 & 0.033 & 0.492 \\
\hline Fisher & $54.16 * * *$ & 1.32 & 1.08 & $77.09 * * *$ & 1.77 & $28.57 * * *$ & 1.43 & $39.04 * * *$ & $14.23 * * *$ & $2.46^{* * *}$ & $2.23 *$ & $15.72 * * *$ \\
\hline Countries & 21 & 14 & 12 & 23 & 23 & 12 & 20 & 15 & 6 & 29 & 28 & 7 \\
\hline Observations & 273 & 180 & 159 & 294 & 308 & 145 & 254 & 199 & 72 & 381 & 360 & 93 \\
\hline
\end{tabular}




\begin{tabular}{|c|c|c|c|c|c|c|c|c|c|c|c|c|}
\hline & \multicolumn{12}{|c|}{ Panel B: Economic globalisation } \\
\hline & LI & MI & Eng. & Frch. & Christ. & Islam & Open & Closed & Oil & Nonoil & Stable & Unstable \\
\hline Constant & $\begin{array}{l}0.241 * * * \\
(0.000)\end{array}$ & $\begin{array}{l}3.45 * * * \\
(0.000)\end{array}$ & $\begin{array}{l}3.724 * * * \\
(0.000)\end{array}$ & $\begin{array}{l}0.284 * * * \\
(0.000)\end{array}$ & $\begin{array}{l}2.017 * * * \\
(0.000)\end{array}$ & $\begin{array}{l}0.349 * * * \\
(0.000)\end{array}$ & $\begin{array}{l}2.553 * * * \\
(0.000)\end{array}$ & $\begin{array}{l}0.249 * * * \\
(0.000)\end{array}$ & $\begin{array}{l}0.376 * * * \\
(0.000)\end{array}$ & $\begin{array}{l}1.712 * * * \\
(0.000)\end{array}$ & $\begin{array}{l}1.834 * * * \\
(0.000)\end{array}$ & $\begin{array}{l}0.262 * * * \\
(0.000)\end{array}$ \\
\hline Economic Glob(IV) & $\begin{array}{l}0.002 * * * \\
\left(0.000^{\circ}\right.\end{array}$ & $\begin{array}{l}0.005 \\
(0.181)\end{array}$ & $\begin{array}{l}0.004 \\
(0.259)\end{array}$ & $\begin{array}{l}0.003 * * * \\
(0.000)\end{array}$ & $\begin{array}{l}\text { 0.004* } \\
(0.061)\end{array}$ & $\begin{array}{l}0.002 * * * \\
(0.000)\end{array}$ & $\begin{array}{l}0.004 * \\
(0.067)\end{array}$ & $\begin{array}{l}0.002 * * * \\
(0.000)\end{array}$ & $\begin{array}{l}0.002 * * * \\
(0.000)\end{array}$ & $\begin{array}{l}0.004 * * \\
(0.027)\end{array}$ & $\begin{array}{l}0.004 * * \\
(0.027)\end{array}$ & $\begin{array}{l}0.002 * * * \\
(0.000)\end{array}$ \\
\hline GDP growth & $\begin{array}{l}0.0006 * \\
(0.072)\end{array}$ & $\begin{array}{l}0.002 \\
(0.794)\end{array}$ & $\begin{array}{l}0.002 \\
(0.783)\end{array}$ & $\begin{array}{l}0.00006 \\
(0.859)\end{array}$ & $\begin{array}{l}0.001 \\
(0.655)\end{array}$ & $\begin{array}{l}-0.0002 \\
(0.546)\end{array}$ & $\begin{array}{l}0.0008 \\
(0.882)\end{array}$ & $\begin{array}{l}0.0009 * \\
(0.062)\end{array}$ & $\begin{array}{l}-0.00008 \\
(0.755)\end{array}$ & $\begin{array}{l}0.001 \\
(0.668)\end{array}$ & $\begin{array}{l}0.001 \\
(0.768)\end{array}$ & $\begin{array}{l}0.0007 * \\
(0.081)\end{array}$ \\
\hline Foreign aid & $\begin{array}{l}-0.0003 * \\
(0.077)\end{array}$ & $\begin{array}{l}-0.0007 \\
(0.956)\end{array}$ & $\begin{array}{l}0.002 \\
(0.831)\end{array}$ & $\begin{array}{l}-0.0001 \\
(0.382)\end{array}$ & $\begin{array}{l}-0.0001 \\
(0.925)\end{array}$ & $\begin{array}{l}-0.001 \\
(0.153)\end{array}$ & $\begin{array}{l}-0.0004 \\
(0.859)\end{array}$ & $\begin{array}{l}-0.0001 \\
(0.745)\end{array}$ & $\begin{array}{l}-0.0005 \\
(0.194)\end{array}$ & $\begin{array}{l}-0.0002 \\
(0.894)\end{array}$ & $\begin{array}{l}0.0002 \\
(0.951)\end{array}$ & $\begin{array}{l}-0.0001 \\
(0.574)\end{array}$ \\
\hline Public Investment & $\begin{array}{l}0.003 * * * \\
(0.000)\end{array}$ & $\begin{array}{l}-0.003 \\
(0.638)\end{array}$ & $\begin{array}{l}-0.007 \\
(0.563)\end{array}$ & $\begin{array}{l}0.003 * * * \\
(0.000)\end{array}$ & $\begin{array}{l}-0.001 \\
(0.799)\end{array}$ & $\begin{array}{l}0.001 * * \\
(0.015)\end{array}$ & $\begin{array}{l}-0.002 \\
(0.653)\end{array}$ & $\begin{array}{l}0.003 * * * \\
(0.002)\end{array}$ & $\begin{array}{l}0.001 * * * \\
(0.006)\end{array}$ & $\begin{array}{l}-0.0006 \\
(0.906)\end{array}$ & $\begin{array}{l}-0.0001 \\
(0.980)\end{array}$ & $\begin{array}{l}0.0008 \\
(0.552)\end{array}$ \\
\hline Inflation & $-0.000 * *$ & -0.003 & -0.000 & -0.000 & -0.000 & $0.0009 * *$ & -0.00005 & $\begin{array}{l}-0.000 * \\
(0.053)\end{array}$ & $\begin{array}{l}0.0006 * * \\
(0.018)\end{array}$ & $\begin{array}{l}-0.000 \\
(0.755)\end{array}$ & $\begin{array}{l}-0.0008 \\
(0.697)\end{array}$ & $\begin{array}{l}\mathbf{0 . 0 0 0 * * *} \\
(\mathbf{0 . 0 0 5})\end{array}$ \\
\hline Adjusted $\mathrm{R}^{2}$ (within) & 0.353 & 0.017 & 0.014 & 0.434 & 0.014 & 0.474 & 0.016 & 0.229 & 0.749 & 0.015 & 0.016 & 0.414 \\
\hline Fisher & $26.30 * * *$ & 0.56 & 0.40 & $39.41 * * *$ & 0.83 & $21.52 * * *$ & 0.72 & $10.68 * * *$ & $34.71 * * *$ & 1.10 & 1.07 & $11.45 * * *$ \\
\hline Countries & 20 & 13 & 12 & 21 & 23 & 10 & 18 & 15 & 5 & 28 & 26 & 7 \\
\hline Observations & 266 & 176 & 159 & 283 & 308 & 134 & 243 & 199 & 68 & 374 & 346 & 93 \\
\hline
\end{tabular}

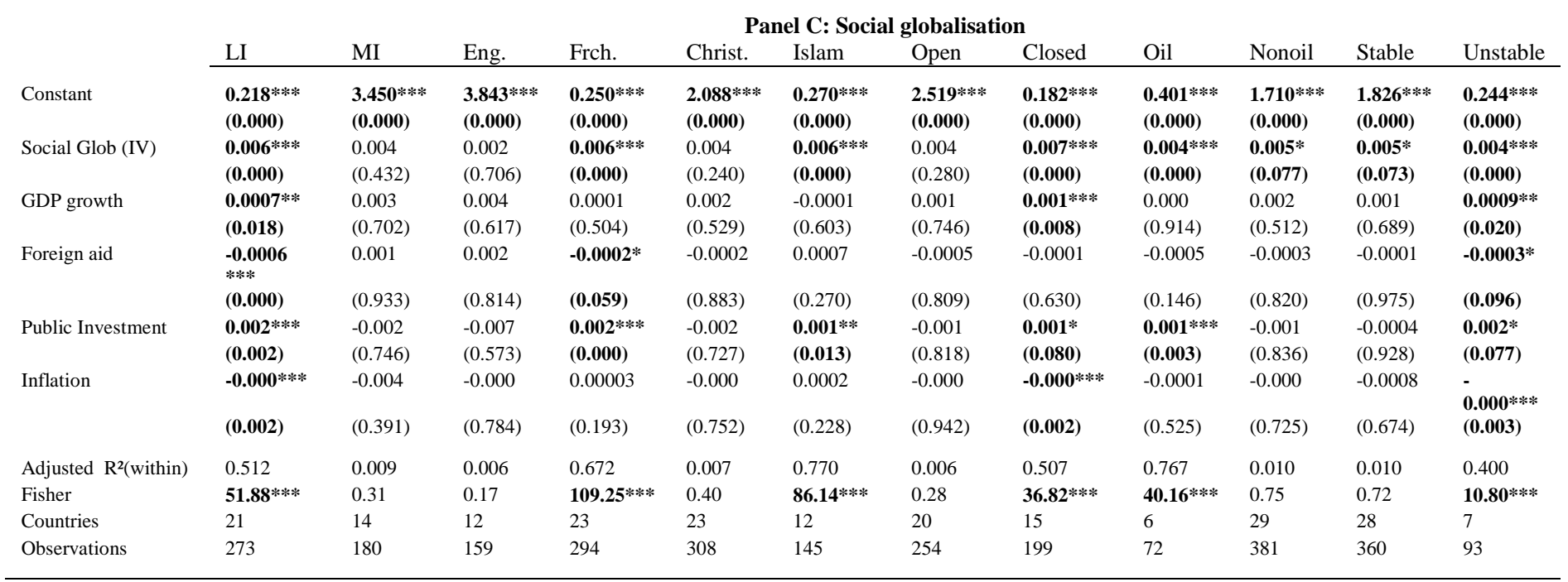

\begin{tabular}{|c|c|c|c|c|c|c|c|c|c|c|c|c|}
\hline & \multicolumn{12}{|c|}{ Panel D: Globalisation } \\
\hline & LI & MI & Eng. & Frch. & Christ. & Islam & Open & Closed & Oil & Nonoil & Stable & Unstable \\
\hline Constant & $\begin{array}{l}0.186 * * * \\
(0.000)\end{array}$ & $\begin{array}{l}3.167 * * * * \\
(0.000)\end{array}$ & $\begin{array}{l}3.538 * * * \\
(0.000)\end{array}$ & $\begin{array}{l}0.217 * * * \\
(0.000)\end{array}$ & $\begin{array}{l}1.927 * * * * \\
(0.000)\end{array}$ & $\begin{array}{l}0.223 * * * \\
(\mathbf{0 . 0 0 0})\end{array}$ & $\begin{array}{l}2.320 * * * \\
(\mathbf{0 . 0 0 0 )}\end{array}$ & $\begin{array}{l}0.168 * * * \\
(0.000)\end{array}$ & $\begin{array}{l}0.356 * * * \\
(0.000)\end{array}$ & $\begin{array}{l}1.573 * * * \\
(0.000)\end{array}$ & $\begin{array}{l}1.672 * * * * \\
(0.000)\end{array}$ & $\begin{array}{l}0.214 * * * \\
(0.000)\end{array}$ \\
\hline Globalisation(IV) & $\begin{array}{l}0.004 * * * \\
(0.000)\end{array}$ & $\begin{array}{l}0.009 * \\
(0.050)\end{array}$ & $\begin{array}{l}0.008 \\
(0.102)\end{array}$ & $\begin{array}{l}0.005 * * * \\
(0.000)\end{array}$ & $\begin{array}{l}0.007 * * * \\
(0.009)\end{array}$ & $\begin{array}{l}0.005 * * * \\
(0.000)\end{array}$ & $\begin{array}{l}0.007 * * \\
(0.018)\end{array}$ & $\begin{array}{l}0.005 * * * \\
(0.000)\end{array}$ & $\begin{array}{l}0.003 * * * \\
(0.000)\end{array}$ & $\begin{array}{l}0.007 * * * \\
(0.001)\end{array}$ & $\begin{array}{l}0.007 * * * \\
(0.002)\end{array}$ & $\begin{array}{l}0.003 \text { *** } \\
(\mathbf{0 . 0 0 0 )}\end{array}$ \\
\hline GDP growth & $\begin{array}{l}0.0006 * * \\
(0.031)\end{array}$ & $\begin{array}{l}0.003 \\
(0.712)\end{array}$ & $\begin{array}{l}0.002 \\
(0.779)\end{array}$ & $\begin{array}{l}0.0001 \\
(0.460)\end{array}$ & $\begin{array}{l}0.001 \\
(0.706)\end{array}$ & $\begin{array}{l}0.00005 \\
(0.843)\end{array}$ & $\begin{array}{l}0.0009 \\
(0.869)\end{array}$ & $\begin{array}{l}0.0008 * * \\
(0.019)\end{array}$ & $\begin{array}{l}0.0003 * \\
(0.071)\end{array}$ & $\begin{array}{l}0.001 \\
(0.731)\end{array}$ & $\begin{array}{l}0.0004 \\
(0.909)\end{array}$ & $\begin{array}{l}0.001 \text { *** } \\
(0.003)\end{array}$ \\
\hline Foreign aid & $\begin{array}{l}-0.0003 \text { ** } \\
(0.033)\end{array}$ & $\begin{array}{l}0.0004 \\
(0.976)\end{array}$ & $\begin{array}{l}0.003 \\
(0.774)\end{array}$ & $\begin{array}{l}-0.0002 * \\
(0.063)\end{array}$ & $\begin{array}{l}-0.0002 \\
(0.906)\end{array}$ & $\begin{array}{l}0.0007 \\
(0.243)\end{array}$ & $\begin{array}{l}-0.0005 \\
(0.824)\end{array}$ & $\begin{array}{l}0.00007 \\
(0.852)\end{array}$ & $\begin{array}{l}- \\
0.0007 * * \\
(0.031)\end{array}$ & $\begin{array}{l}-0.0001 \\
(0.915)\end{array}$ & $\begin{array}{l}0.0004 \\
(0.899)\end{array}$ & $\begin{array}{l}-0.0002 \\
(0.204)\end{array}$ \\
\hline Public Investment & $\begin{array}{l}0.001 * * \\
(0.019)\end{array}$ & $\begin{array}{l}-0.004 \\
(0.572)\end{array}$ & $\begin{array}{l}-0.007 \\
(0.546)\end{array}$ & $\begin{array}{l}0.001 * * * \\
(0.000)\end{array}$ & $\begin{array}{l}-0.003 \\
(0.574)\end{array}$ & $\begin{array}{l}0.0007 * \\
(0.085)\end{array}$ & $\begin{array}{l}-0.003 \\
(0.578)\end{array}$ & $\begin{array}{l}0.001 \\
(0.133)\end{array}$ & $\begin{array}{l}0.001 * * * \\
(0.000)\end{array}$ & $\begin{array}{l}-0.002 \\
(0.603)\end{array}$ & $\begin{array}{l}-0.001 \\
(0.699)\end{array}$ & $\begin{array}{l}0.0007 \\
(0.524)\end{array}$ \\
\hline Inflation & $\begin{array}{l}-0.000 * * * \\
(0.001)\end{array}$ & -0.003 & -0.000 & -0.000 & -0.000 & $\begin{array}{l}0.0007 * * * \\
(0.002)\end{array}$ & $\begin{array}{l}-0.00004 \\
(0.917)\end{array}$ & $\begin{array}{l}-0.000 * * * \\
(0.002)\end{array}$ & $\begin{array}{l}0.0002 \\
(0.200)\end{array}$ & $\begin{array}{l}-0.000 \\
(0.697)\end{array}$ & $\begin{array}{l}-0.001 \\
(0.614)\end{array}$ & $\begin{array}{l}- \\
\mathbf{0 . 0 0 0 * * *} \\
(\mathbf{0 . 0 0 1 )}\end{array}$ \\
\hline Adjusted $\mathrm{R}^{2}$ (within) & 0.615 & 0.029 & 0.023 & 0.748 & 0.026 & 0.794 & 0.025 & 0.565 & 0.837 & 0.030 & 0.031 & 0.558 \\
\hline Fisher & $79.09 * * *$ & 0.97 & 0.69 & $157.89 * * *$ & 1.50 & $98.71 * * *$ & 1.18 & $46.64 * * *$ & $62.86 * * *$ & $2.19 * * *$ & $2.09 *$ & $20.50 * * *$ \\
\hline Countries & 21 & 14 & 12 & 23 & 23 & 12 & 20 & 15 & 6 & 29 & 28 & 7 \\
\hline Observations & 273 & 180 & 159 & 294 & 308 & 145 & 254 & 199 & 72 & 381 & 360 & 93 \\
\hline
\end{tabular}

$*, * *, * * *$ : significance levels of $10 \%, 5 \%$ and $1 \%$ respectively. IV: Instrumented Variable. Glob: Globalisation. LI: Low Income. MI: Middle Income. Eng. English common law. Frch: French civil law. Christ: Christian-dominated. Islam: Islam-oriented. Open: Unlandlocked. Closed: Landlocked. Oil: petroleum exporting. Nonoil: Non petroleum exporting. Stable: Politically stable. Unstable: Politically unstable. 
Table 3: Comparative economics with Instrumental Variable Tobit regressions

\begin{tabular}{|c|c|c|c|c|c|c|c|c|c|c|c|c|}
\hline & \multicolumn{12}{|c|}{ Panel A: Political globalisation } \\
\hline & \multicolumn{2}{|c|}{ Income levels } & \multicolumn{2}{|c|}{ Legal origins } & \multicolumn{2}{|l|}{ Religion } & \multicolumn{2}{|c|}{ Openness to sea } & \multicolumn{2}{|c|}{ Oil exports } & \multicolumn{2}{|c|}{ Political stability } \\
\hline & LI & MI & Eng. & Frch. & Christ. & Islam & Open & Closed & Oil & Nonoil & Stable & Unstable \\
\hline Constant & $\begin{array}{l}0.318 * * * \\
(0.000)\end{array}$ & $\begin{array}{l}4.289 \\
(0.282)\end{array}$ & $\begin{array}{l}-2.347 \\
(0.572)\end{array}$ & $\begin{array}{l}0.294 * * * \\
(0.000)\end{array}$ & $\begin{array}{l}-0.844 \\
(0.696)\end{array}$ & $\begin{array}{l}0.450 * * * \\
(0.000)\end{array}$ & $\begin{array}{l}2.998 \\
(0.335)\end{array}$ & $\begin{array}{l}0.479 * * * \\
(0.000)\end{array}$ & $\begin{array}{l}0.350 * * * \\
(\mathbf{0 . 0 0 0})\end{array}$ & $\begin{array}{l}2.204 \\
(0.226)\end{array}$ & $\begin{array}{l}0.218 * * * \\
(0.000)\end{array}$ & $\begin{array}{l}3.203 \\
(0.109)\end{array}$ \\
\hline Political Glob (IV) & $\begin{array}{l}\text { 0.001*** } \\
(\mathbf{0 . 0 0 1 )}\end{array}$ & $\begin{array}{l}0.073 \\
(0.100)\end{array}$ & $\begin{array}{l}\mathbf{0 . 1 8 9 * * * *} \\
(\mathbf{0 . 0 0 1 )}\end{array}$ & $\begin{array}{l}0.002 * * * \\
(0.000)\end{array}$ & $\begin{array}{l}0.102 * * * \\
(0.001)\end{array}$ & $\begin{array}{l}0.001 * * * \\
(0.000)\end{array}$ & $\begin{array}{l}0.038 \\
(0.344)\end{array}$ & $\begin{array}{l}-\mathbf{0 . 0 0 2} * * * \\
(\mathbf{0 . 0 0 0 )}\end{array}$ & $\begin{array}{l}0.002 * * * \\
(\mathbf{0 . 0 0 4 )}\end{array}$ & $\begin{array}{l}0.039 \\
(0.103)\end{array}$ & $\begin{array}{l}\mathbf{0 . 0 0 2} * * * \\
(\mathbf{0 . 0 0 0 )}\end{array}$ & $\begin{array}{l}0.035 \\
(0.165)\end{array}$ \\
\hline GDP growth & $\begin{array}{l}0.003 * * * \\
(0.003)\end{array}$ & $\begin{array}{l}-0.238 \\
(0.488)\end{array}$ & $\begin{array}{l}0.043 \\
(0.863)\end{array}$ & $\begin{array}{l}0.0006 \\
(0.666)\end{array}$ & $\begin{array}{l}0.172 \\
(0.212)\end{array}$ & $\begin{array}{l}0.0003 \\
(0.795)\end{array}$ & $\begin{array}{l}0.018 \\
(0.931)\end{array}$ & $\begin{array}{l}0.003 * * \\
(0.020)\end{array}$ & $\begin{array}{l}-0.0004 \\
(0.828)\end{array}$ & $\begin{array}{l}0.126 \\
(0.294)\end{array}$ & $\begin{array}{l}\text { 0.005*** } \\
(\mathbf{0 . 0 0 0 )}\end{array}$ & $\begin{array}{l}0.036 \\
(0.800)\end{array}$ \\
\hline Foreign aid & $\begin{array}{l}-0.002 * * * \\
(0.000)\end{array}$ & $\begin{array}{l}- \\
0.868 * * * * \\
(0.002)\end{array}$ & $\begin{array}{l}- \\
0.517 * * * \\
(0.000)\end{array}$ & $-0.006 * * *$ & $\begin{array}{l}-0.160 * * * \\
(0.002)\end{array}$ & $-0.023 * * *$ & $-0.142 * *$ & $\begin{array}{l}-0.005 * * * \\
(0.000)\end{array}$ & $\begin{array}{l}-0.014 \\
* * * \\
(0.000)\end{array}$ & $\begin{array}{l}- \\
\mathbf{0 . 1 3 2} * * * \\
(\mathbf{0 . 0 0 5})\end{array}$ & $\begin{array}{l}- \\
\mathbf{0 . 0 0 1} * * * \\
(\mathbf{0 . 0 0 3})\end{array}$ & $\begin{array}{l}- \\
0.216 * * * \\
(0.001)\end{array}$ \\
\hline Public Investment & 0.001 & $\begin{array}{l}-0.457 * * \\
(0.019)\end{array}$ & -0.241 & $\begin{array}{l}0.003 * * * \\
(0.009)\end{array}$ & $\begin{array}{c}-0.293 * \\
(0.052)\end{array}$ & $\begin{array}{l}0.006 * * * \\
(0.000)\end{array}$ & $\begin{array}{l}-0.301 * \\
(0.052)\end{array}$ & $\begin{array}{l}0.008 * * * \\
(0.000)\end{array}$ & $\begin{array}{l}0.010 * * * \\
(0.000)\end{array}$ & $\begin{array}{l}-0.298 * * \\
(0.021)\end{array}$ & $\begin{array}{l}0.001 \\
(0.591)\end{array}$ & $\begin{array}{l}0.314 * * * \\
(0.007)\end{array}$ \\
\hline Inflation & $-0.010 * *$ & 0.166 & -0.0002 & $\begin{array}{l}-0.0003 \\
* * * \\
(0.008)\end{array}$ & -0.0001 & -0.001 & -0.014 & -0.000 & -0.0007 & -0.0001 & $\begin{array}{l}- \\
\mathbf{0 . 0 0 0 * * *} \\
(0.007)\end{array}$ & 0.088 \\
\hline LR Chi-Square & $50.43 * * *$ & $18.89 * * *$ & $27.40 * * *$ & $192.54 * * *$ & $28.71 * * *$ & $255.67 * * *$ & $11.65 * *$ & $79.65 * * *$ & $72.80 * * *$ & $22.45 * * *$ & $45.34 * * *$ & $27.46 * * *$ \\
\hline Log Likelihood & 315.841 & -680.078 & -604.221 & 265.399 & -1091.346 & 183.124 & -928.278 & 206.736 & 73.713 & -1318.43 & 123.828 & -1251.97 \\
\hline Observations & 273 & 180 & 159 & 294 & 308 & 145 & 254 & 199 & 72 & 381 & 93 & 360 \\
\hline
\end{tabular}

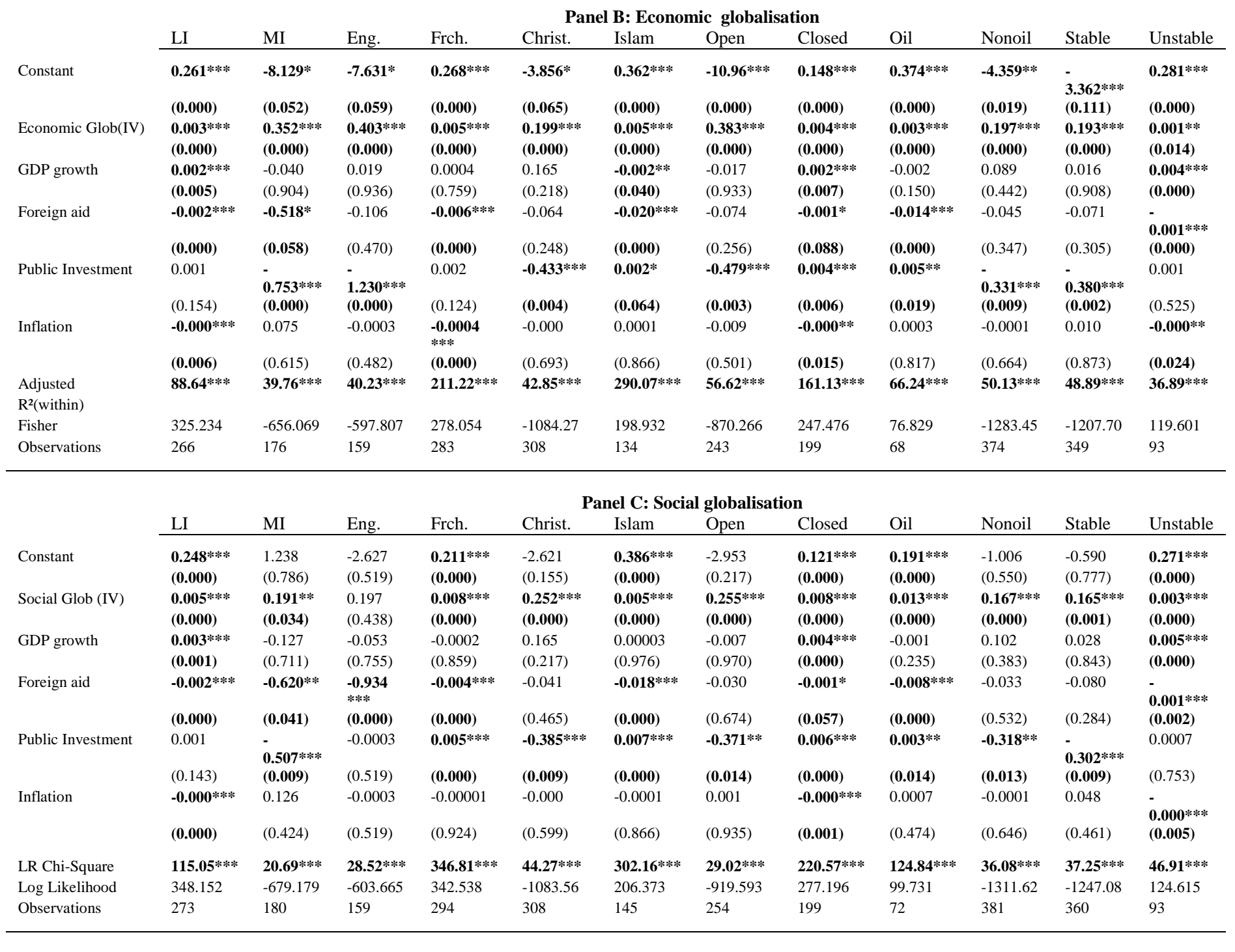




\begin{tabular}{|c|c|c|c|c|c|c|c|c|c|c|c|c|}
\hline & \multicolumn{12}{|c|}{ Panel D: Globalisation } \\
\hline & LI & MI & Eng. & Frch. & Christ. & Islam & Open & Closed & Oil & Nonoil & Stable & Unstable \\
\hline Constant & $\begin{array}{l}0.205 * * * \\
(0.000)\end{array}$ & $\begin{array}{l}- \\
19.06 * * * * \\
(0.002)\end{array}$ & $\begin{array}{l}- \\
21.70 * * * \\
(0.000)\end{array}$ & $\begin{array}{l}0.108 * * * \\
(0.000)\end{array}$ & $\begin{array}{l}- \\
10.972 * * * \\
(0.000)\end{array}$ & $\begin{array}{l}0.276 * * * \\
(0.000)\end{array}$ & $\begin{array}{l}0.141 * * * \\
(0.000)\end{array}$ & $\begin{array}{l}- \\
13.497 * * * \\
(0.000)\end{array}$ & $\begin{array}{l}0.091 \\
(0.125)\end{array}$ & $\begin{array}{l}- \\
7.202 * * * \\
(0.202)\end{array}$ & $\begin{array}{l}- \\
9.108 * * * \\
(0.002)\end{array}$ & $\begin{array}{l}0.232 * * * \\
(0.000)\end{array}$ \\
\hline Globalisation(IV) & $\begin{array}{l}0.004 * * * \\
(0.000)\end{array}$ & $\begin{array}{l}0.531 * * * \\
(0.000)\end{array}$ & $\begin{array}{l}0.692 * * * \\
(0.000)\end{array}$ & $\begin{array}{l}0.008 * * * \\
(0.000)\end{array}$ & $\begin{array}{l}0.370 * * * \\
(0.000)\end{array}$ & $\begin{array}{l}0.006 * * * \\
(0.000)\end{array}$ & $\begin{array}{l}0.005 * * * \\
(0.000)\end{array}$ & $\begin{array}{l}0.410 * * * \\
(0.000)\end{array}$ & $\begin{array}{l}0.010 * * * \\
(0.000)\end{array}$ & $\begin{array}{l}0.258 * * * \\
(0.000)\end{array}$ & $\begin{array}{l}0.305 * * * \\
(0.000)\end{array}$ & $\begin{array}{l}0.003 * * * \\
(0.000)\end{array}$ \\
\hline GDP growth & $\begin{array}{l}0.002 * * * \\
(0.003)\end{array}$ & $\begin{array}{l}-0.056 \\
(0.862)\end{array}$ & $\begin{array}{l}0.134 \\
(0.563)\end{array}$ & $\begin{array}{l}-0.0001 \\
(0.900)\end{array}$ & $\begin{array}{l}0.113 \\
(0.386)\end{array}$ & $\begin{array}{l}0.0002 \\
(0.865)\end{array}$ & $\begin{array}{l}0.003 * * * \\
(0.005)\end{array}$ & $\begin{array}{l}-0.014 \\
(0.943)\end{array}$ & $\begin{array}{l}0.0001 \\
(0.903)\end{array}$ & $\begin{array}{l}0.047 \\
(0.683)\end{array}$ & $\begin{array}{l}-0.021 \\
(0.875)\end{array}$ & $\begin{array}{l}0.005 * * * \\
(0.000)\end{array}$ \\
\hline Foreign aid & $\begin{array}{l}-0.001 * * * \\
(0.000)\end{array}$ & $\begin{array}{l}-0.348 \\
(0.215)\end{array}$ & $\begin{array}{l}-0.058 \\
(0.673)\end{array}$ & $\begin{array}{l}-0.004 * * * \\
(0.000)\end{array}$ & $\begin{array}{l}-0.011 \\
(0.839)\end{array}$ & $\begin{array}{l}-0.018 * * * \\
(0.000)\end{array}$ & $\begin{array}{l}-0.002 * * * \\
(0.000)\end{array}$ & $\begin{array}{l}-0.003 \\
(0.965)\end{array}$ & $\begin{array}{l}-0.011 * * * \\
(0.000)\end{array}$ & $\begin{array}{l}0.0002 \\
(0.997)\end{array}$ & $\begin{array}{l}-0.005 \\
(0.944)\end{array}$ & $\begin{array}{l}0.001 * * * * \\
(0.002)\end{array}$ \\
\hline Public Investment & $\begin{array}{l}0.0009 \\
(0.462)\end{array}$ & $\begin{array}{l}- \\
0.579 * * * \\
(0.002)\end{array}$ & $-1.00 * * *$ & $0.003 * * *$ & $-0.410 * * *$ & $0.006 * * *$ & $0.004 * *$ & $\begin{array}{l}-0.432 * * * \\
(0.003)\end{array}$ & $\begin{array}{l}0.005 * * * \\
(0.001)\end{array}$ & $\begin{array}{l}- \\
\mathbf{0 . 3 3 5 * * *} \\
(\mathbf{0 . 0 0 8 )}\end{array}$ & $\begin{array}{l}- \\
0.323 * * * \\
(0.005)\end{array}$ & $\begin{array}{l}0.001 \\
(0.153)\end{array}$ \\
\hline Inflation & $\begin{array}{l}-0.000 * * * \\
(0.001)\end{array}$ & $\begin{array}{l}0.292 * \\
(0.060)\end{array}$ & $\begin{array}{l}-0.0003 \\
(0.403)\end{array}$ & $\begin{array}{l}-0.0002 * \\
(0.083)\end{array}$ & $\begin{array}{l}-0.0002 \\
(0.498)\end{array}$ & $\begin{array}{l}0.0005 \\
(0.575)\end{array}$ & $\begin{array}{l}-0.000 * * * \\
(0.009)\end{array}$ & $\begin{array}{l}0.002 \\
(0.841)\end{array}$ & $\begin{array}{l}0.002 \\
(0.110)\end{array}$ & $\begin{array}{l}-0.0001 \\
(0.559)\end{array}$ & $\begin{array}{l}0.005 \\
(0.933)\end{array}$ & $\begin{array}{l}- \\
\mathbf{0 . 0 0 0 * * * *} \\
(\mathbf{0 . 0 0 8 )}\end{array}$ \\
\hline LR Chi-Square & $103.51 * * *$ & $39.74 * * *$ & $54.12 * * *$ & $311.61 * * *$ & $60.49 * * *$ & $309.36 * * *$ & $111.13 * * *$ & $43.25 * * *$ & $106.69 * * *$ & $48.99 * * *$ & $52.82 * * *$ & $45.43 * * *$ \\
\hline Log Likelihood & 342.384 & -669.651 & -590.860 & 324.935 & -1075.45 & 209.970 & 222.476 & -912.477 & 90.659 & -1305.16 & -1239.30 & 123.874 \\
\hline Observations & 273 & 180 & 159 & 294 & 308 & 145 & 199 & 254 & 72 & 381 & 360 & 93 \\
\hline
\end{tabular}

*,**,***: significance levels of 10\%, 5\% and 1\% respectively. IV: Instrumented Variable. Glob: Globalisation. LI: Low Income. MI: Middle Income. Eng. English common law. Frch: French civil law. Christ: Christian-dominated. Islam: Islam-oriented. Open: Unlandlocked. Closed: Landlocked. Oil: petroleum exporting. Nonoil: Non petroleum exporting. Stable: Politically stable. Unstable: Politically unstable.

\subsection{Further discussion of results and policy implications}

The positive nexus between globalisation and inclusive development can be elicited from the perspective that the two measurements have theoretical bases that are founded on the efficient and optimal allocation and/distribution of resources. On the one hand, the measure for inclusive development appreciates how three principal achievements (income, education and health) within an economy are distributed among the population, by taking into account inequality. On the other hand, the neoliberal school on globalisation discussed in Section 2 is based on the imperative of optimal allocation of global resources for economic development.

The established positive connection is in accordance with Firebaugh (2004) who has advocated that the phenomenon of globalisation is tailored to lift developing countries out of poverty and enhance their human development standards. On a lighter note, the findings do not broadly align with the conclusions from indirect assessments by Asongu et al (2015) and Azzimonti et al. (2014).

It is also important to devote space to elucidating the puzzle of resource-rich countries experiencing low levels of inclusive human development compared with their resource-poor counterparts. Such clarification can be from stylized facts on the one hand and on the Fosu conjectures on the other. From the perspective of stylized facts, average growth in African countries has been driven primarily by price booms in natural resources (especially in petroleum) during the period of study. Unfortunately, most of these resource-rich countries have been associated with comparatively lower degrees of inclusive development from the 
health and social perspectives. For instance, consistent with Ndikumana and Boyce (2012), Equatorial Guinea, Gabon and the Republic of Congo are among the most wealthy countries in the African continent with (i) per capita incomes of respectively $\$ 8,649\left(2^{\text {nd }}\right), \$ 4,176\left(5^{\text {th }}\right)$ and $\$ 1,253\left(15^{\text {th }}\right)$ and (ii) substantial reserves in oil with Gabon ranking $7^{\text {th }}$, the Congo Republic ranking $8^{\text {th }}$ and Equatorial Guinea ranking $10^{\text {th }}$. This is in sharp contrast to the inclusive human development levels enjoyed by these countries because most citizens are living in poverty. Accordingly, these citizens lack drinkable water, health care, decent sanitation and elementary schools. The second and third to the last in terms of immunization against measles are Gabon and Equatorial Guinea with 55 percent and 51 percent rates respectively. Moreover, the odds of a child reaching his/her fifth birthday in Equatorial Guinea are higher than the African average. Recent rankings on quality of growth from Mlachila et al. (2014, p.17) confirm these formalized ideas. Such rankings show that inclusive development has been deteriorating in the three resource-rich countries. The performance of these three countries in a sample of 93 developing nations from 1990-1994, 1995-1999, 20002004 and 2005-2011 shows a considerable degradation in inclusive development: Congo Republic $\left(59^{\text {th }}, 70^{\text {th }}, 74^{\text {th }} \& 84^{\text {th }}\right)$; Equatorial Guinea $\left(76^{\text {th }}, 73^{\text {rd }}, 76^{\text {th }} \& 88^{\text {th }}\right)$ and Gabon $\left(58^{\text {th }}\right.$, $\left.61^{\text {st }}, 67^{\text {th }} \& 69^{\text {th }}\right)$.

In the light of the above, it is not surprising that the growth experienced by these resource-wealthy countries is not trickling down to the poor. This can be explained by the Fosu conjectures. In essence, exclusive development can be elucidated from the perspective that the response of poverty to growth is a decreasing function of inequality. This is also due to the fact that economic growth is globalisation-driven. Income distribution is fundamental in the effect of growth on poverty (Fosu, 2015a; Fosu, 2011). This is consistent with the view that inclusiveness plays a fundamental role in the poverty-growth nexus (Fosu, 2015a; Fosu, 2010b). In more specific terms: "The study finds that the responsiveness of poverty to income is a decreasing function of inequality" (Fosu, 2010c, p. 818); “The responsiveness of poverty to income is a decreasing function of inequality, and the inequality elasticity of poverty is actually larger than the income elasticity of poverty" (Fosu, 2010a, p. 1432) and "In general, high initial levels of inequality limit the effectiveness of growth in reducing poverty while growing inequality increases poverty directly for a given level of growth" (Fosu, 2011, p. 11). It follows that the recent growth resurgence has not benefited African countries because of low initial levels of inclusive human development or high initial levels of inequality. 
The findings in this study have overwhelmingly confirmed the tested hypotheses. It follows that for each fundamental characteristic, one sub-panel is more of a policy syndrome in the post-2015 sustainable development agenda. Accordingly, more resources need to be devoted to support: low income, French civil law, landlocked, Islam-oriented, oil-rich and politically unstable countries.

For these policy syndromes ${ }^{3}$, domestic as well as foreign policies could be tailored towards addressing binding constraints to an improved globalisation-inclusiveness relationship. Such binding constraints could be the lack of financial resources needed for human capability development, provision of public commodities and social mobility among others. Given the apparent issues of governance in inclusive development, such financial resources would need to be aligned more for the improvement of economic governance which is the formulation and implementation of measures that enable an effective delivery of public commodities such as education and health facilities.

\section{Conclusions and future research directions}

This study has examined the impact of globalisation on inclusive human development in 51 African countries for the period 1996-2011, with particular emphasis on income levels (low income versus middle income), legal origins (English common law versus French civil law), resource wealth (oil-rich versus oil-poor), landlockedness (landlocked versus unlandlocked), religious domination (Christianity versus Islam) and political stability (stable versus unstable). The empirical evidence is based on instrumental variable panel Fixed effects and Tobit regressions in order to control for the unobserved heteroegeneity and limited range in the dependent variable. Political, economic, social and general globalisation variables are used. Six main hypotheses are investigated. The findings broadly show that middle income, English common law, oil-poor, unlandlocked, Christian-oriented and politically-stable countries are associated with comparatively high levels of globalisation-driven inclusive human development. Puzzling findings are clarified and policy implications discussed.

\footnotetext{
${ }^{3}$ Whereas Fosu (2013a) has defined policy syndromes as situations that are detrimental to growth in Africa, Asongu (2015c) has conceived policy syndromes as fundamental characteristics needing more resources in order to achieve a particular target. The conception of policy syndrome in this study is consistent with both authors because the identified fundamental characteristics are comparatively more detrimental to enjoying the inclusive benefits of globalisation and hence, more resources are needed to support them.
} 
It is important to highlight that whereas indicators on African development may be of poor quality and unreliable, to the best of our knowledge those from the World Bank and United Nations Development Program are comparatively less unreliable. In essence, low capacity and lack of funding are the main drivers of data gap in the continent (Beegle et al., 2016).

Future studies can improve the tie between globalisation and inclusive human development by investigating the relationship throughout the conditional distributions of inclusive human development. The motivation for this future direction is that blanket policies based on mean effects may not succeed unless they are contingent on initial values of inclusive development and hence tailored differently across countries with low, intermediate and high initial levels of inclusive development. 


\section{Appendices}

\section{Appendix 1: Definitions of Variables}

\begin{tabular}{|c|c|c|c|}
\hline Variables & Signs & Definitions of variables (Measurement) & Sources \\
\hline $\begin{array}{l}\text { Inclusive human } \\
\text { development }\end{array}$ & IHDI & Inequality Adjusted Human Development Index & UNDP \\
\hline $\begin{array}{l}\text { Political } \\
\text { Globalisation }\end{array}$ & Polglob & $\begin{array}{l}\text { "This captures the extent of political globalisation in terms of } \\
\text { number of foreign embassies in a country, membership in } \\
\text { international orgnisations, participation in UN security". }\end{array}$ & Dreher et al. (2010) \\
\hline $\begin{array}{l}\text { Economic } \\
\text { Globalisation }\end{array}$ & Ecoglob & $\begin{array}{l}\text { "Overall economic globalisation (considers both the flow and } \\
\text { the restrictions in a given country to derive this). The higher, } \\
\text { the better social globalisation". }\end{array}$ & Dreher et al. (2010) \\
\hline $\begin{array}{l}\text { Social } \\
\text { Globalisation }\end{array}$ & Socglob & $\begin{array}{l}\text { "Overall scores for the countries extent of social } \\
\text { globalisation. The higher the better socially globalised the } \\
\text { country". }\end{array}$ & Dreher et al. (2010) \\
\hline Globalisation & Glob & $\begin{array}{l}\text { This is an overall index that contains economic globalisation, } \\
\text { social globalisation and political globalisation }\end{array}$ & Dreher et al. (2010) \\
\hline GDP growth & GDPg & Gross Domestic Product (GDP) growth (annual \%) & World Bank (WDI) \\
\hline Foreign aid & Aid & Total Development Assistance (\% of GDP) & World Bank (WDI) \\
\hline Public Investment & Pub. Ivt. & Gross Public Investment (\% of Grosss) & World Bank (WDI) \\
\hline Inflation & Inflation & Annual Consumer Price Inflation & World Bank (WDI) \\
\hline
\end{tabular}

WDI: World Bank Development Indicators. UNDP: United Nations Development Program.

Appendix 2: Summary statistics (1996-2011)

\begin{tabular}{lrrrrr}
\hline & Mean & SD & Minimum & Maximum & Observations \\
\cline { 2 - 5 } Inclusive Human Development & 1.521 & 6.926 & 0.127 & 0.809 & 553 \\
Political Globalisation (IV) & 58.696 & 17.576 & 22.439 & 93.575 & 765 \\
Economic Globalisation (IV) & 44.991 & 12.643 & 14.041 & 84.229 & 645 \\
Social Globalisation (IV) & 28.865 & 11.113 & 6.582 & 65.004 & 765 \\
Globalisation (IV) & 41.775 & 9.881 & 18.774 & 68.453 & 756 \\
Education(SSE) & 40.941 & 26.892 & 4.022 & 123.893 & 491 \\
Mobile phone penetration & 19.829 & 29.390 & 0.000 & 171.515 & 811 \\
GDP growth & 4.863 & 7.297 & -32.832 & 106.279 & 792 \\
Population growth & 2.317 & 1.007 & -1.081 & 9.770 & 816 \\
Foreign aid & 10.212 & 12.245 & -0.251 & 147.054 & 791 \\
Public Investment & 7.491 & 4.692 & 0.000 & 43.011 & 713 \\
Inflation & 54.723 & 925.774 & -9.797 & 24411.03 & 717 \\
\hline S.D: Standard Deviation. & & & & &
\end{tabular}


Appendix 2: Correlation matrix (uniform sample size: 442)

\begin{tabular}{|c|c|c|c|c|c|c|c|c|c|}
\hline IVPolglob & IVEcoglob & IVSocglob & IVGlob & GDPg & Aid & Pub.Ivt. & Inflation & IHDI & \\
\hline \multirow[t]{9}{*}{1.000} & 0.062 & 0.276 & 0.604 & 0.011 & -0.211 & 0.043 & 0.022 & 0.118 & IVPolglob \\
\hline & 1.000 & 0.643 & 0.769 & -0.006 & -0.329 & 0.066 & -0.0003 & 0.297 & IVEcoglob \\
\hline & & 1.000 & 0.854 & -0.058 & -0.471 & -0.029 & 0.016 & 0.273 & IVSocglob \\
\hline & & & 1.000 & -0.022 & -0.452 & 0.038 & 0.017 & 0.310 & IVGlob \\
\hline & & & & 1.000 & 0.198 & 0.276 & -0.113 & -0.026 & GDPg \\
\hline & & & & & 1.000 & 0.209 & -0.002 & -0.170 & Aid \\
\hline & & & & & & 1.000 & -0.082 & -0.128 & Pub. Ivt. \\
\hline & & & & & & & 1.000 & -0.011 & Inflation \\
\hline & & & & & & & & 1.000 & IHDI \\
\hline
\end{tabular}

IV: Instrumented value. Polgov: Political Globalisation. Ecoglob: Economic Globalisation. Socglob: Social Globalisation. Glob: Globalisation. SSE: Secondary School Enrolment. Mobile: Mobile Phone Penetration. GDPg: Gross Domestic Product growth. Popg: Population growth. Aid: Foreign aid. Pub. Ivt: Public Investment. IHDI: Inequality Adjusted Human Development Index.

\section{Appendix 4: Categorization of Countries}

Catego
Income

levels

Low Income

Income

Ghana, Guinea, Guinea-Bissau, Kenya, Liberia, Madagascar, Malawi, Mali,

Mauritania, Mozambique, Niger, Rwanda, Sierra Leone, Somalia, Togo, Uganda,

Zambia, Zimbabwe.

English Botswana, The Gambia, Ghana, Kenya, Lesotho, Liberia, Malawi, Mauritius,

Common-law Namibia, Nigeria, Seychelles, Sierra Leone, Somalia, South Africa, Sudan,

Legal

Origins

Swaziland, Uganda, Zambia, Zimbabwe.

Algeria, Angola, Benin, Burkina Faso, Burundi, Cameroon, Cape Verde, Central African Republic, Chad, Comoros, Congo Democratic Republic, Congo Republic,

French Civil- Côte d'Ivoire, Djibouti, Egypt, Equatorial Guinea, Eritrea, Ethiopia, Gabon, Guinea, law Guinea-Bissau, Libya, Madagascar, Mali, Mauritania, Morocco, Mozambique, Niger, Rwanda, Senegal, Togo, Tunisia.

Christianity Angola, Benin, Botswana, Burundi, Cameroon, Cape Verde, Central African Republic, Congo Democratic Republic, Congo Republic, Côte d'Ivoire, Equatorial

Religion Guinea, Eritrea, Ethiopia, Gabon, Ghana, Kenya, Lesotho, Liberia, Madagascar, Malawi, Mauritius, Mozambique, Namibia, Rwanda, Seychelles, South Africa, South Africa, Togo, Uganda, Zambia, Zimbabwe.

Islam Algeria, Burkina Faso, Chad, Comoros, Djibouti, Egypt, The Gambia, Guinea, Guinea Bissau, Libya, Mali, Mauritania, Morocco, Niger, Nigeria, Senegal, Sierra Leone, Somalia, Sudan, Tunisia,

Petroleum Algeria, Angola, Cameroon, Chad, Congo Republic, Equatorial Guinea, Gabon,

Exporting Libya, Nigeria, Sudan.

Resources

Benin, Botswana, Burkina Faso, Burundi, Cape Verde, Central African Republic, Comoros, Congo Democratic Republic, Côte d'Ivoire, Djibouti, Eritrea, Ethiopia,

Non- $\quad$ Egypt, The Gambia, Ghana, Guinea, Guinea-Bissau, Kenya, Lesotho, Liberia,

Petroleum Madagascar, Malawi, Mali, Mauritania, Mauritius, Morocco, Mozambique,

Exporting Namibia, Niger, Senegal, Sierra Leone, Somalia, Rwanda, Seychelles, South Africa, Swaziland, Togo, Tunisia, Uganda, Zambia, Zimbabwe.

Conflict

Angola, Burundi, Chad, Central African Republic, Congo Democratic Republic, 
Non-Conflict Senegal, Rwanda, Seychelles, South Africa, Swaziland, Togo, Tunisia, Uganda, Zambia.

Landlocked Botswana, Burkina Faso, Burundi, Chad, Central African Republic, Ethiopia,
Lesotho, Malawi, Mali, Niger, Rwanda, Swaziland, Uganda, Zambia, Zimbabwe

Openness to Sea

Not landlocked
Algeria, Angola, Benin, Cameroon, Cape Verde, Comoros, Congo Democratic Republic, Congo Republic, Côte d'Ivoire, Djibouti, Egypt, Equatorial Guinea, Eritrea, Gabon, The Gambia, Ghana, Guinea, Guinea-Bissau, Kenya, Liberia, Libya, Madagascar, Mauritania, Mauritius, Morocco, Mozambique, Namibia, Nigeria, Senegal, Sierra Leone, Somalia, Sudan, Seychelles, South Africa, Togo, Tunisia.

Num: Number of cross sections (countries)

\section{References}

Agbor, J. A. (2015). "How does colonial origin matter for economic performance in subSaharan Africa?", In Augustin K. Fosu (Ed.), Growth and Institutions in African Development, Chapter 13, pp. 309-327, Routledge Studies in Development Economics: New York.

Amavilah, V. H. (2015). Social Obstacles to Technology, Technological Change, and the Economic Growth of African Countries: Some Anecdotal Evidence from Economic History, MPRA Paper No. 63273, Munich.

America, R., (2013). "Economic Development with Limited Supplies of Management. What to do about it - the case of Africa", Challenge, 56(1), pp. 61-71.

Anand, R., Mishra, S., \& Spatafora, N., (2012), "Structural Transformation and the Sophistication of Production," IMF Working Paper No. 12/59, Washington.

Anyanwu, J., \& Erhijakpor, A., (2014). "Does Oil Wealth Affect Democracy in Africa?"African Development Review, 26 (1), pp. 15-37.

Ariss, R. T. (2010). "On the Implications of Market Power in Banking: Evidence from Developing Countries", Journal of Banking and Finance, 34(4), pp. 765-775.

Arvis, J-F., Marteau, J-F., \& Raballand, G. (2007). The cost of being landlocked: logistics costs and supply chain reliability", Word Bank Working Paper Series No. 4258, Washington.

Asongu, S.A. (2013), "Globalization and Africa: implications for human development", International Journal of Development Issues, 12(3), pp. 213-238.

Asongu, S. A. (2014a). "Financial development dynamic thresholds of financial globalisation: evidence from Africa”, Journal of Economics Studies, 41(2), pp. 166-195. 
Asongu, S. A., (2014b). "Globalisation (fighting) corruption and development. How are these phenomena linearly and non-linearly related in wealth effects?", Journal of Economic Studies, 41(3), pp. 346-369.

Asongu, S. A., (2014c). "African development: beyond income convergence", South African Journal of Economics, 82(3), pp. 334-353.

Asongu, S. A., (2015a). "Reinventing foreign aid for inclusive and sustainable development:

Kuznets, Piketty and the great policy reversal", Journal of Economic Surveys:

http://onlinelibrary.wiley.com/doi/10.1111/joes.12109/abstract

Asongu, S. A., (2015b). "Law, Finance, Economic Growth and Welfare: Why Does Legal Origin Matter?”, Institutions and Economies, 7(2), pp. 30-55.

Asongu, S. A., (2015c). "Knowledge Economy Gaps, Policy Syndromes, and Catch-Up Strategies: Fresh South Korean Lessons to Africa", Journal of Knowledge Economy, http://link.springer.com/article/10.1007/s13132-015-0321-0

Asongu, S. A., Efobi, U., \& Breecroft, I., (2015). "Inclusive Human Development in Precrisis Times of Globalization-driven Debts", African Development Review, 27(4), pp. 428-442

Asongu, S. A., \& De Moor, L., (2016). "Financial globalisation dynamic thresholds for financial development: evidence from Africa", The European Journal of Development Research. DOI: 10.1057/ejdr.2016.10.

Asongu, S. A., \& Nwachukwu, J., (2015a). "Revolution empirics: predicting the Arab Spring” Empirical Economics: http://link.springer.com/article/10.1007/s00181-015-1013-0

Asongu, S. A., \& Nwachukwu, J. C., (2016a). "Foreign aid and inclusive development: updated evidence from Africa, 2005-2012”, Social Science Quarterly: DOI:

$10.1111 /$ ssqu. 12275 .

Asongu, S. A., \& Nwachukwu, J. C., (2016b). "The Role of Governance in Mobile Phones for Inclusive Human Development in Sub-Saharan Africa”, Technovation, 55-56 (SeptemberOctober), pp. 1-13.

Asongu, S. A., \& Nwachukwu, J. C., (2016c). "Finance and Inclusive Human Development: Evidence from Africa", Brussels Economic Review: Forthcoming.

Atkinson, A. B., Piketty, T., Saez, E. (2011). "Top Incomes in the Long Run of History”, Journal of Economics Literature, 49(1), pp. 3-71.

Azzimonti, M., De Francisco, E., \& Quadrini, V. (2014). "Financial Globalisation, Inequality and the Rising Public Debt”, American Economic Review, 104(8), pp. 2267-2302.

Beck, T., Demirgüç-Kunt, A., \& Levine, R. (2003). Law and finance: why does legal origin matter?, Journal of Comparative Economics, 31(4), 653-675. 
Beegle, K., Christiaensen, L., Dabalen, A., \& Gaddis, I., (2016). "Poverty in a Rising Africa", Africa Poverty Report, the World Bank, Washington.

http://www.worldbank.org/en/region/afr/publication/poverty-rising-africa-poverty-report

(Accessed: 23/07/2016).

Carson, R. T., \& Sun, Y. (2007). “The Tobit model with a non-zero threshold”, Econometrics Journal, 10(3), pp. 488-502.

CIA (2011). Central Intelligence Agency, The World Factbook 2011.

Coccorese, P., \& Pellecchia, A. (2010). "Testing the 'Quiet Life' Hypothesis in the Italian Banking Industry”, Economic Notes by Banca dei Paschi di Siena SpA, 39(3), pp. 173-202.

Dreher, A., Gaston, N., Martens, P., \& Van Boxem, L., (2010). "Measuring Globalization Opening the Black Box. A Critical Analysis of Globalization Indices", Journal of Globalization Studies, 1(1), pp. 166-185.

Efobi, U., (2015). "Politicians' Attributes and Institutional Quality in Africa: A Focus on Corruption", Journal of Economic Issues, 49(3), pp. 787-813.

Fields, G., (2015). “Aid, Growth and Jobs”, African Development Review, Supplement: Special Issue on "Aid and Employment", 27,( S1), pp. 5-16.

Firebaugh, G., (2004), "Accounting for the recent decline in global income inequality", American Journal of Sociology, 110(2), pp. 283-312.

Fonchingong, C., (2014). "Firming Up Institutional Policy for Deprived Elderly in Cameroon", Politics \& Policy, 42(6), pp. 948-980.

Fosu, A. K., (2015a). "Growth, Inequality and Poverty in Sub-Saharan Africa: Recent Progress in a Global Context”, Oxford Development Studies, 43(1), pp. 44-59.

Fosu, A., (2015b). Growth and Institutions in African Development, First edited by Augustin K. Fosu, , Routledge Studies in Development Economics: New York

Fosu, A., (2015c). Growth and institutions in African Development, in Growth and Institutions in African Development, First edited by Augustin K. Fosu, 2015, Chapter 1, pp. 1-17, Routledge Studies in Development Economics: New York.

Fosu, A., (2013a), "Growth of African Economies: Productivity, Policy Syndromes and the Importance of Institutions" Journal of African Economies, 22(4), pp. 523-551.

Fosu, A. (2013b). Achieving development success: Strategies and lessons from the developing world, UNU-WIDER Policy Brief (November), Helsinki.

Fosu, A. K. (2008). "Inequality and the Growth-Poverty Nexus: Specification Empirics Using African Data", Applied Economics Letters, 15(7), pp. 563-566.

Fosu, A. K. (2009). "Inequality and the Impact of Growth on Poverty: Comparative Evidence for Sub-Saharan Africa”, Journal of Development Studies, 45(5), pp. 726-745. 
Fosu, A. K. (2010a). "Inequality, Income and Poverty: Comparative Global Evidence", Social Sciences Quarterly, 91(5), pp. 1432-1446.

Fosu, A. K. (2010b). "The Effect of Income Distribution on the Ability of Growth to Reduce Poverty: Evidence from Rural and Urban African Economies", American Journal of Economics and Sociology, 69(3), pp. 1034-1053.

Fosu, A. K. (2010c). "Does Inequality Constrain Poverty Reduction Programs? Evidence from Africa", Journal of Policy Modeling, 32(6), pp. 818-827.

Fosu, A. K., (2011). "Growth, Inequality and Poverty Reduction in Developing Countries: Recent Global Evidence”, UNU WIDER Working Paper 2011/01, Helsinki.

Grennes, T. (2003), “Creative destruction and globalization”, Cato Journal, 22(3), pp. 543-558.

Jones, S., Page, J., Shimeles, A., \& Tarp, F., (2015). “Aid, Growth and Employment in Africa", African Development Review, Supplement: Special Issue on "Aid and Employment", 27, ( S1), pp. 1-4.

Jones, S., \& Tarp, F., (2015). "Priorities for Boosting Employment in Sub-Saharan Africa: Evidence for Mozambique", African Development Review, Supplement: Special Issue on "Aid and Employment", 27,( S1), pp. 56-70.

Kenneth, R., \& Himes, O. F. M., (2008). "Globalization with a Human Face: Catholic Social Teaching and Globalization”, Theological Studies, 69(2), pp. 269-289.

Kose, M. A., Prasad, E. S., Rogoff, K., \& Wei, S. J. (2006). "Financial globalization: a reappraisal", IMF Staff Papers 56(1), pp. 8-62.

Kose, M. A., Prasad, E. S., \& Taylor, A. D. (2011). “Threshold in the process of international financial integration”, Journal of International Money and Finance 30(1), pp.147-179.

Koetter, M., Kolari, J. W., \& Spierduk, L. (2008). Efficient Competition ? Testing the 'Quiet Life' of U.S Banks with Adjusted Lerner Indices, Proceedings of the $44^{\text {th }}$ 'Bank Structure and Competition' Conference, Federal Reserve Bank of Chicago.

Kuada, J. (2015). Private Enterprise-Led Economic Development, In J. Kuada (Ed.), SubSaharan Africa The Human Side of Growth, Palgrave Macmillan: New York.

Kumbhakar, S. C., \& Lovell, C. A. K. (2000). Stochastic Frontier Analysis, Cambridge MA: Cambridge University Press.

La Porta, R., Lopez-de-Silanes, F., Shleifer, A., \& Vishny, R. W. (1998). "Law and finance”, Journal of Political Economy, 106(6), 1113-1155.

La Porta, R., Lopez-de-Silanes, F., Shleifer, A., \& Vishny, R. W. (1999). "The quality of government", Journal of Law, Economics and Organization, 15(1), pp. 222-279. 
La Porta, R., Lopez-de-Silanes, F., \& Shleifer, A., (2008), “The Economic Consequences of Legal Origin," Journal of Economic Literature, 46(2), pp. 285-332.

Lalountas, D.A., Manolas, G.A., \& Vavouras, I. S., (2011), “ Corruption, globalization and development: How are these three phenomena related?", Journal of Policy Modeling, 33(4), pp. 636-648.

Leautier, F. A., (2012). "What Role for Africa After 50 Years of Independence: Provider of Natural Resources or a New Global Leader?", Journal of African Development, 14(1), pp. 127-151.

Lewis, A. (1955). Theory of Economic Growth. Milton Park: Routledge.

McDonald, J. (2009). "Using Least Squares and Tobit in Second Stage DEA Efficiency Analyses", European Journal of Operational Research, 197(2), pp.792-798.

Mishra, S., Gable, S. L., \& Anand, R., (2011), "Service Export Sophsitication and Economic Growth,” World Bank Policy Working Paper No. 5606, Washington.

Mlachila, M., Tapsoba, R., \& Tapsoba, S. J. A., (2014). “A Quality of Growth Index for Developing Countries: A Proposal”, IMF Working Paper No. 14/172, Washington.

Mthuli, N., Anyanwu, J. C., \& Hausken, K. (2014). "Inequality, Economic Growth and Poverty in the Middle East and North Africa (MENA)", African Development Review, 26(3), pp. 435-453.

Ndikumana, L., \& Boyce, J. K., (2012) "Rich Presidents of Poor Nations: Capital Flight from Resource-Rich Countries in Africa", Department of Economics and Political Economy Research Institute (PERI), University of Massachusetts Amherst. http://concernedafricascholars.org/wp-content/uploads/2012/11/caploss01-ndiku-14th.pdf (Accessed: 28/12/2014).

Obeng-Odoom, F. (2015). "Africa: On the Rise, but to Where?", Forum for Social Economics, 44(3), pp. 234-250.

Page, J., \& Shimeles, A., (2015). "Aid, Employment and Poverty Reduction in Africa", African Development Review, Supplement: Special Issue on "Aid and Employment", 27,(S1), pp. 17-30.

Page, J., \& Söderbom, M., (2015). "Is Small Beautiful? Small Enterprise, Aid and Employment in Africa", African Development Review, Supplement: Special Issue on "Aid and Employment", 27,(S1), pp. 44-55.

Pesaran, M. H., (2006). "Estimation and inference in large heterogeneous panels with a multifactor error structure". Econometrica 74(4), pp. 967-1012.

Petras, J., \& Veltmeyer, H., (2001), Globalization Unmasked: Imperialism in the 21st Century,Zed Books, London. 
Piketty, T. (2014). “Capital in the Twenty First Century”, Harvard University Press.

Pinkivskiy, M., \& Sala-i-Martin, X., (2014). “Africa is on time”, Journal of Economic Growth, 19(3), pp. 311-333.

Price, G. N., \& Elu, J. U. (2014). "Does regional currency integration ameliorate global macroeconomic shocks in sub-Saharan Africa? The case of the 2008-2009 global financial crisis", Journal of Economic Studies, 41(5), pp. 737-750.

Rodrik, D., Subramanian, A. \& Trebbi, F., (2004), "Institution rule: the primacy of institutions over geography and integration in economic development", Journal of Economic Growth, 9(2), pp. 131-165.

Roudometof, V., (2014). "Religion and globalisation", in The SAGE Handbook of Globalisation, Edited by Steger, M., Battersby, P., \& Siracusa, J., Chapter 10, pp. 151-165, SAGE Publications: London.

Scholte, J. A. (2000), Globalisation: A Critical Introduction, St. Martin's Press Inc, New York, NY.

Seneviratne, D., \& Sun, Y., (2013), "Infrastructure and Income Distribution in ASEAN-5: What are the Links?" IMF Working Paper No. 13/41, Washington.

Simpasa, A, Shimeles, A., \& Salami, A. O., "Employment Effects of Multilateral Development Bank Support: The Case of the African Development Bank", African Development Review, Supplement: Special Issue on "Aid and Employment", 27, ( S1), pp. 3143.

Sirgy, M.J., Lee, D.J., Miller, C., \& Littlefield, J. E., (2004), "The impact of globalization on a country's quality of life: toward an integrated model”, Social Indictors Research, 68(3) pp. 251-298.

Smart, B. (2003), Economy, Culture and Society: A Sociological Critique of Neo-liberalism, Open Univesity Press, Buckingham.

Stiglitz, J. (2000). "Capital Market Liberalization, Economic Growth and Instability”, World Development, 28(6), pp. 1075-1086.

Tchamyou, V. S., (2015). "The role of knowledge economy in African business", African Governance and Development Institute Working Paper No. 15/049, Yaoundé.

Tobin, J. (1958). "Estimation of relationships for limited dependent variables". Econometrica 26(1), pp. 24-36.

Tsai, M. (2006), Does Globalisation Affect Human Well-being? Department of Sociology, National Taipei University, Taiwan. 
United Nations (2013). "A New Global Partnership: Eradicate Poverty and Transform Economies Through Sustainable Development", The Report of the High-Level Panel of Eminent Persons on the Post-2015 Development Agenda.

http://www.un.org/sg/management/pdf/HLP_P2015_Report.pdf (Accessed: 07/12/2014).

World Bank (2015). "World Development Indicators", World Bank Publications http://www.gopa.de/fr/news/world-bank-release-world-development-indicators-2015

(Accessed: 25/04/2015). 